\title{
PARTICLE METHOD FOR THE SOLUTION OF STOCHASTIC PROBLEMS: FORMULATION
}

\author{
R. Reyes Sotomayor ${ }^{1,2}$, A. F. Sarmiento ${ }^{1}$, D. A. García ${ }^{1}$, J.M. Mantilla ${ }^{1}$, D. A. Garzón- \\ Alvarado ${ }^{1}$, E. Patiño ${ }^{1}$ \\ ${ }^{1}$ Department of Mechanical and Mechatronics Engineering, National University of Colombia. \\ ${ }^{2}$ Corresponding author (rreyess@unal.edu.co).
}

\begin{abstract}
This work presents the initial approach to a novel method for numerical solution of stochastic differential equations, showing the proper formulation for the stochastic term in a Lagrangian method. The mathematical formulation for the uncertainty properties terms of the model is based in the Karhunen-Loeve expansions used in the spectral stochastic finite element method (SSFEM). The particle method used is the Smoothed Particle Hydrodynamics (SPH), which is modified to represent the randomness of the output variables that are affected by the stochastic inlet properties behavior. This method formulation acquires importance for the solution of high deformation problems where there exists an uncertainty on the model properties.
\end{abstract}

Keywords: Smoothed Particle Hydrodynamics, Stochastic Spectral Methods, Stochastic Differential Equation.

\section{INTRODUCTION}

The stochastic spectral approach has a lot of advantages in the problems when the uncertainty of some properties is an important factor in the behavior of the real phenomena[13]. Nowadays the stochastic spectral scheme is being implemented in the finite element method successfully to represent the randomness of the results in structural and transport problems[4-6], the aim of formulating an spectral stochastic scheme in a Lagrangian particle method is the main capacity to simulate high deformation, mobile boundary and interface problems[7-9]. The stochastic spectral smoothed particle hydrodynamics method (SSSPH) may have diverse application in the development of models that need the benefits of the particle methods and want to obtain results based on reliability.

The SSSPH method represents the system by a number of particles that have the material properties and interact according to a fixed volume defined by a kernel function [10], [11], this properties are represented by the Karhunen-Loeve expansion when they act randomly [12-14]. The representation of these properties as a polynomial expansion must have an impact on the response variable of the simulation, which are represented by a chaos polyno- 
mial expansion [3], [15], [16]. The solution of the continuity equations is obtained locally for each particle.

This work presents a basic formulation for the conduction problem using the Stochastic Spectral Smoothed Particle Hydrodynamics (SSSPH) method and an application example showing the stochastic solutions for the temperature conduction problem.

\section{SPH METHOD}

A little summary of the SPH method is presented here, for more details see [9]. The basis of SPH is interpolation theory, and its formulation involves two steps. In the first step the representation of a function in its continuous form is done through the use of a kernel estimate of the field variables at a point [17], this approximation of the function is based on the evaluation of the smoothing kernel function. Then, the second step is the representation of the interpolation kernel over the defined domain using discrete variables [8].

The interpolated value of a function $f$ in the space defined by $\vec{x}$ vector can be expressed using SPH smoothing as (1).

$$
\left\langle f\left(\vec{x}_{i}\right)\right\rangle=\sum_{j=1}^{N} \frac{m_{j}}{\rho_{j}} f_{j} W\left(\vec{x}_{i}-\vec{x}_{j}, h\right)
$$

Where $m_{j}$ and $\rho_{j}$ are the mass and density of particle $j, W\left(\vec{x}_{i}-\vec{x}_{j}, h\right)$ is the smoothing kernel, $\mathrm{h}$ is the smoothing length (see more in [18]) and $N$ is the total number of particles. The gradient of function $f$ can also be found by differentiating the interpolation (1) as expressed in (2).

$$
\left\langle\nabla f\left(\vec{x}_{i}\right)\right\rangle=\sum_{j=1}^{N} \frac{m_{j}}{\rho_{j}} f_{j} \nabla_{i} W\left(\vec{x}_{i}-\vec{x}_{j}, h\right)
$$

Definition of the kernel function is represented in (3).

$$
W\left(\vec{x}_{i}-\vec{x}_{j}, h\right)=\frac{1}{h^{d}} \varphi\left(\frac{\left\|\vec{x}_{i}-\vec{x}_{j}\right\|}{h}\right)=W_{i j}
$$

Where $\varphi$ is the function that describes the kernel approximation and $d$ is the number of space dimensions. Although there are several smoothing functions widely used, it is usually preferred the cubic B-spline [10][11], which is defined by (4). 


$$
W(q, h)=\alpha_{d} \begin{cases}\frac{2}{3}-q^{2}+\frac{1}{2} q^{3} & 0 \leq q<1 \\ \frac{1}{6}(2-q)^{3} & 1 \leq q<2 \\ 0 & 2 \leq q\end{cases}
$$

Where $\alpha_{d}$ is the constant of normalization, which depends on the number of spatial dimensions (for one dimension is $\alpha_{1}=1 / h$, for two dimensions is $\alpha_{2}=15 / 7 \pi h^{2}$ and for three dimensions is $\left.\alpha_{3}=3 / 2 \pi h^{3}\right), q=\| \vec{x}_{i}-\vec{x}_{j}|| / h$ and $\left\|\vec{x}_{i}-\vec{x}_{j}\right\|$ is the distance between two particles.

\section{SPECTRAL STOCHASTIC METHOD}

Usually the numerical simulations of many phenomena are based in a deterministic scheme; this kind of simulations does not consider the variability of some properties in the real behavior of physical processes. In some cases the representation of the phenomenon without including the variability of the properties is enough to understand and simulate the process, but when the behavior of the properties and field variables is uncertain and it is desire to have a high reliability in the results it is necessary to use a stochastic representation of physical phenomena.

To predict the behavior of a process with high variability a stochastic scheme could be used, with this the properties are not modeled by its mean value only, but in a range of possible values defined by its probabilistic distribution. Then it is possible to obtain a better simulation of the phenomena than with a deterministic scheme, because it delivers a range where the behavior of the process could occur. There are several stochastic schemes that have been used including the Monte Carlo Method and the Spectral Stochastic Method [3].

The implementation of the Spectral Stochastic Method begins with the representation of a property with a stochastic process using a covariance function (5), where $K(\theta, x)$ represents the stochastic process, $\operatorname{cov}\left(x_{1}, x_{2}\right)$ represents the covariance function, $x$ denote the space coordinates and $\theta$ the randomness of the process. The covariance function related to the randomness of the property is symmetric and positive defined, its eigenvectors are orthogonal and its eigenvalues are real, so it is possible to represent the stochastic process with the Karhunen-Loeve polynomial (6) [12], [16].

$$
\begin{gathered}
K(\theta, x)=f\left(\operatorname{cov}\left(x_{1}, x_{2}\right), \bar{K}(x)\right) \\
K(\theta, x)=\bar{K}(x)+\sum_{h=1}^{\infty} \sqrt{\lambda_{h}} K_{h}(x) \xi_{h}
\end{gathered}
$$

In the Karhunen-Loeve expansion, $\bar{K}(x)$ is the mean value of the property, $\lambda_{h}$ and $k_{h}(x)$ are the eigenvalues and the eigenvector of the covariance function and $\xi_{h}$ are an independent variable group of a random space.

To obtain the eigenvalues and eigenvectors the covariance function must be evaluated 
in (7) over the domain of the problem $\Omega$. For a square two-dimensional space the covariance function is defined as indicated in (8) [12].

$$
\begin{aligned}
& \int_{\Omega} \operatorname{cov}\left(x_{1}, x_{2} ; y_{1}, y_{2}\right) k_{h}\left(x_{2}, y_{2}\right) d x_{2} d y_{2}=\lambda_{h} k_{h}\left(x_{1}, y_{1}\right) \\
& \operatorname{cov}\left(x_{1}, y_{1} ; x_{2}, y_{2}\right)=C_{0} \exp \left(-\frac{\left|x_{1}-x_{2}\right|}{b_{x}}-\frac{\left|y_{1}-y_{2}\right|}{b_{y}}\right)
\end{aligned}
$$

Where $C_{0}$ represents the variance value, $b_{x}$ and $b_{y}$ represent the correlation lengths. Then the eigenvectors and eigenvalues are defined splitting the function in two terms, one for each coordinate (9).

$$
\begin{aligned}
& k_{h}\left(x_{1}, y_{1}\right)=k_{h_{x}}\left(x_{1}\right) * k_{h_{y}}\left(y_{1}\right) \\
& \lambda_{h}=\lambda_{h_{x}} * \lambda_{h_{y}}
\end{aligned}
$$

Introducing equation (9) in (7) the integral can be solved independently for each dimension [2], [12], obtaining the eigenvalues and eigenvectors in the same way for the two dimensions, given by (10), where $\omega$ terms were calculated by Choi as [2].

$$
\begin{gathered}
\lambda_{h_{x}}=\frac{2 \sqrt{C_{0}}}{b_{x}\left(\left(\frac{1}{b_{x}}\right)^{2}+\omega_{h_{x}}^{2}\right)} \\
k_{h_{x}}\left(x_{1}\right)= \begin{cases}\frac{\cos \left(\frac{\left(h_{x}-1\right) \pi}{2 a} x_{1}\right)}{\sqrt{a+\left(\frac{\sin \left(2 \omega_{h_{x}} a\right)}{2 \omega_{h_{x}}}\right)}} & h_{x} \text { even } \\
\frac{\sin \left(\frac{\left(h_{x}-1\right) \pi}{2 a} x_{1}\right)}{\sqrt{a-\left(\frac{\sin \left(2 \omega_{h_{x}} a\right)}{2 \omega_{h_{x}}}\right)}} & h_{x} \text { odd }\end{cases}
\end{gathered}
$$

To include the influence of the stochastic process in any response variable studied, it is represented in terms of the chaos polynomial base (11) where $\bar{\phi}$ represent the mean value, the summation represents the fluctuation behavior of the variable and $\Psi$ represents the Hermit polynomials, which are also orthogonal [15], [16].

$$
\phi=\bar{\phi}+\sum_{r=1}^{\infty} \phi_{b} \psi_{b}(\xi)
$$


Due to the need to truncate the Karhunen-Loeve and the chaos polynomials expansion up to the Pth term, the error approximation will not be identically zero, so in order to reduce the error, the response variable is projected over the chaos polynomial base (12), this expresses the resulting equation as a function of $\xi$ and $\Psi$ that are grouped in (13) and defined in (14).

$$
\begin{gathered}
\left\langle\phi, \psi_{b}(\xi)\right\rangle=\bar{\phi}+\left(\sum_{b=1}^{P} \phi_{r}^{j} \Psi_{b}(\xi)\right) \cdot \psi_{l}(\xi) \\
\left\langle\xi_{h} \Psi_{b}(\omega) \Psi_{l}(\omega)\right\rangle=\overline{\xi_{h} \Psi_{b}(\omega) \Psi_{l}(\omega)}=B_{h b l} \\
B_{h b l}=\left\{\begin{array}{cc}
\frac{h ! b ! l !}{\left(\frac{h+b-l}{2}\right) !\left(\frac{b+l-h}{2}\right) !\left(\frac{h+l-b}{2}\right) !} & \text { if }(\mathrm{h}+b+l) \text { is even } \wedge l \in[|h-b|, h-b] \\
0 & \text { otherwise }
\end{array}\right.
\end{gathered}
$$

\section{SSSPH IMPLEMENTATION}

The implementation of the SSSPH method begins introducing the Karhunen-Loeve polynomial expansion in the property with randomness characteristics. This polynomial expansion is introduced in the traditional energy continuity equation (15). In this paper the random property selected is the heat conductivity, so equation (16) shows the energy equation with the Karhunen-Loeve polynomial term.

$$
\begin{gathered}
\rho \frac{d u}{d t}=\sigma: d-\nabla \cdot(k \nabla T) \\
\rho \frac{d u}{d t}=\sigma: d-\nabla \cdot\left(\sum_{h=0}^{\infty} k_{h} \xi_{h} \sqrt{\lambda_{h}}\right) \nabla T
\end{gathered}
$$

Where, for the equations (15) and (16), $\rho$ is the density, $u$ the internal energy, $T$ the temperature, $\sigma$ the stress tensor, $d$ the linear strain rate tensor, $k$ the conductivity, and $t$ the time

The next step on the implementation holds the discretization of the expanded energy equation (17). The discretization of the thermal conduction part of equation using SPH is done following the method described by Cleary [19]. The stress and linear strain rate tensors are discretized using the traditional SPH method explained in part 2.

$$
\frac{d u_{i}}{d t}=\sum_{j=1}^{N}\left[\frac{1}{2} m_{j}\left(\frac{\sigma_{i}}{\rho_{i}^{2}}+\frac{\sigma_{j}}{\rho_{j}^{2}}\right): d-\frac{2 m_{j}\left(\sum_{h=0}^{\infty} k_{h} \xi_{h} \sqrt{\lambda_{h}}\right)_{j} T_{i j}}{\rho_{i} \rho_{j}} \frac{x_{i j} \cdot \nabla W_{i j}}{r_{i j}^{2}}\right]
$$


To prevent unphysical results in the heat diffusion, an artificial term is added. This artificial term is based on the total energy, the internal and the kinetic energy dissipation, as shown in equation (18) where the correction parameters $\alpha$ and $\alpha_{u}$, and the signal velocities $v_{\text {sig }}$ and $v_{\text {sig }}^{u}$ are defined as Price [20].

$$
\left(\frac{d u_{i}}{d t}\right)_{d i s s}=-\sum_{j=1}^{N} \frac{m_{j}}{\rho_{i}}\left(\frac{1}{2} \alpha v_{s i g}\left(v_{i j} \cdot r_{i j}\right)^{2}+\alpha_{u} v_{s i d}^{u}\left(u_{i}-u_{j}\right)\right) x_{i j} \cdot \nabla W_{i j}
$$

The artificial diffusion term is added to the energy equation (17) as follows in (19).

$$
\frac{d u_{i}}{d t}=\sum_{j=1}^{N}\left[\frac{1}{2} m_{j}\left(\frac{\sigma_{i}}{\rho_{i}^{2}}+\frac{\sigma_{j}}{\rho_{j}^{2}}\right): d-\frac{2 m_{j}\left(\sum_{h=0}^{\infty} k_{h} \xi_{h} \sqrt{\lambda_{h}}\right)_{j} T_{i j}}{\rho_{i} \rho_{j}} \frac{x_{i j} \cdot \nabla W_{i j}}{r_{i j}^{2}}\right]+\left(\frac{d u_{i}}{d t}\right)_{d i s s}
$$

In order to represent the influence of the stochastic process in the response variable, the chaos polynomials expansion is introduced in the left hand of the discretized equation, truncated up to the Pth term as shown in (20).

$$
\left(\sum_{b=0}^{P} \frac{d u_{b}}{d t} B_{0 b l}\right)_{i}=\sum_{j=1}^{N}\left[\frac{1}{2} m_{j}\left(\frac{\sigma_{i}}{\rho_{i}^{2}}+\frac{\sigma_{j}}{\rho_{j}^{2}}\right): d-\frac{2 m_{j} \sum_{b=0}^{P} \sum_{h=0}^{P} k_{h j} \sqrt{\lambda_{h j}} T_{b i j} B_{h b l}}{\rho_{i} \rho_{j}} \frac{x_{i j} \cdot \nabla W_{i j}}{r_{i j}^{2}}\right]+\left(\frac{d u_{i}}{d t}\right)_{d i s s}
$$

The example problems have both Dirichlet and Neumann boundary conditions. The Dirichlet boundary conditions are implemented by avoiding the temporal update over the particles in this boundary, the Neumann condition is implemented by adding the discretized heat flux term in equation (18) as shown in equation (21), where $q_{j}$ is the heat flux vector.

$$
\frac{d u_{i}}{d t}=\sum_{j=1}^{N}\left[\frac{1}{2} m_{j}\left(\frac{\sigma_{i}}{\rho_{i}^{2}}+\frac{\sigma_{j}}{\rho_{j}^{2}}\right): d-\frac{2 m_{j}\left(\sum_{h=0}^{\infty} k_{h} \xi_{h} \sqrt{\lambda_{h}}\right)_{j} T_{i j}}{\rho_{i} \rho_{j}} \frac{x_{i j} \cdot \nabla W_{i j}}{r_{i j}^{2}}+\frac{m_{j}}{\rho_{j}} q_{j} \cdot \nabla W_{i j}\right]+\left(\frac{d u_{i}}{d t}\right)_{d i s s}
$$

Rearranging the equations the problem results in a linear system problem showed in equations (21) to (23).

$$
A_{b l j}=-\frac{2 m_{j}}{\rho_{i} \rho_{j}} \frac{x_{i j} \cdot \nabla W_{i j}}{r_{i j}^{2}} \sum_{h=0}^{M} k_{h j} \sqrt{\lambda_{h j}} B_{h b l}
$$




$$
\begin{gathered}
B_{000} \frac{d u_{0}}{d t}+B_{010} \frac{d u_{1}}{d t}+B_{020} \frac{d u_{2}}{d t}+\cdots+B_{0 P 0} \frac{d u_{P}}{d t}=\sum_{j=1}^{N}\left(A_{00 j} T_{i j 0}+A_{10 j} T_{i j 1}+A_{20 j} T_{i j 2}+\cdots+A_{P 0 j} T_{i j P}\right)+\sum_{j=1}^{N}\left(\frac{1}{2} m_{j}\left(\frac{\sigma_{i}}{\rho_{i}^{2}}+\frac{\sigma_{j}}{\rho_{j}^{2}}\right): d\right) \\
B_{001} \frac{d u_{0}}{d t}+B_{011} \frac{d u_{1}}{d t}+B_{021} \frac{d u_{2}}{d t}+\cdots+B_{0 P 1} \frac{d u_{P}}{d t}=\sum_{j=1}^{N}\left(A_{01 j} T_{i j 0}+A_{11 j} T_{i j 1}+A_{21 j} T_{i j 2}+\cdots+A_{P 1 j} T_{i j P}\right) \\
\vdots \\
B_{000} \frac{d u_{0}}{d t}+B_{015} \frac{d u_{1}}{d t}+B_{02 s} \frac{d u_{2}}{d t}+\cdots+B_{0 P s} \frac{d u_{P}}{d t}=\sum_{j=1}^{N}\left(A_{00 j} T_{i j 0}+A_{1 j j} T_{i j 1}+A_{2 s j} T_{i j 2}+\cdots+A_{P s j} T_{i j P}\right) \\
\vdots \\
B_{00 P} \frac{d u_{0}}{d t}+B_{01 P} \frac{d u_{1}}{d t}+B_{02 P} \frac{d u_{2}}{d t}+\cdots+B_{0 P P} \frac{d u_{P}}{d t}=\sum_{j=1}^{N}\left(A_{0 P j} T_{i j 0}+A_{1 P j} T_{i j 1}+A_{2 P j} T_{i j 2}+\cdots+A_{P P j} T_{i j P}\right)
\end{gathered}
$$

The stress energy terms are added in the deterministic part of the equation (22), due to the absence of stochastic behavior on their properties. Any other deterministic term, such as heat generation, could be added in the same part.

$$
\underbrace{\left(\begin{array}{ccc}
B_{000} & \cdots & B_{0 P 0} \\
\vdots & \ddots & \vdots \\
B_{00 P} & \cdots & B_{0 P P}
\end{array}\right)}_{\underline{\underline{B}}} \underbrace{\left(\begin{array}{c}
\frac{d u_{0}}{d t} \\
\vdots \\
\frac{d u_{P}}{d t}
\end{array}\right)_{i}}_{\underline{\underline{\underline{d T}}}}=\sum_{j=1}^{N}\left(\begin{array}{ccc}
A_{00} & \cdots & A_{P 0} \\
\vdots & \ddots & \vdots \\
A_{0 P} & \cdots & A_{P P}
\end{array}\right)_{j}\left(\begin{array}{c}
T_{0} \\
\vdots \\
T_{P}
\end{array}\right)_{i j}
$$

The solution is done for each particle as traditionally in SPH problems. The predictorcorrector scheme is used to update the variables over the time [21].

\section{RESULTS}

The SSSPH method is implemented in two thermal conduction problems with different boundary conditions for a square domain. The first model has Dirichlet boundary conditions, one boundary is set to $100^{\circ} \mathrm{C}$ and the others at $20^{\circ} \mathrm{C}$. The second model has 3 Dirichlet boundary conditions set at $20^{\circ} \mathrm{C}$ and one Neumann boundary condition set as an inlet heat flux equal to 10 Watts. All the particles are fixed and the temperature initial condition is set at $20^{\circ} \mathrm{C}$ for both problems.

Results for the test problems for a minute of simulation are shown in figures of Table 1 and 2, where the mean and up to the fourth stochastic variables are shown every 15 seconds. Table 1 shows the results for test problem using the Dirichlet boundaries and Table 2 for the test problem using Neumann and Dirichlet boundaries.

As seen in Table 1 and Table 2, mean temperature shows the standard behavior for a Dirichlet and Neumann problems, decreasing its value in every frame when increasing in $\mathrm{x}$ dimension and diffusing over the plate longitude along the time. The stochastic variables shows similar behavior as the mean value, showing diffusion along the time, but only the first mode shows the same symmetric behavior as the mean value respect to the $\mathrm{x}$ axis, the other modes tend to a mirrored relation in its values respect to the $\mathrm{x}$ axis. 
Table 1. Deterministic and stochastic solutions for the Dirichlet boundary heat problem.

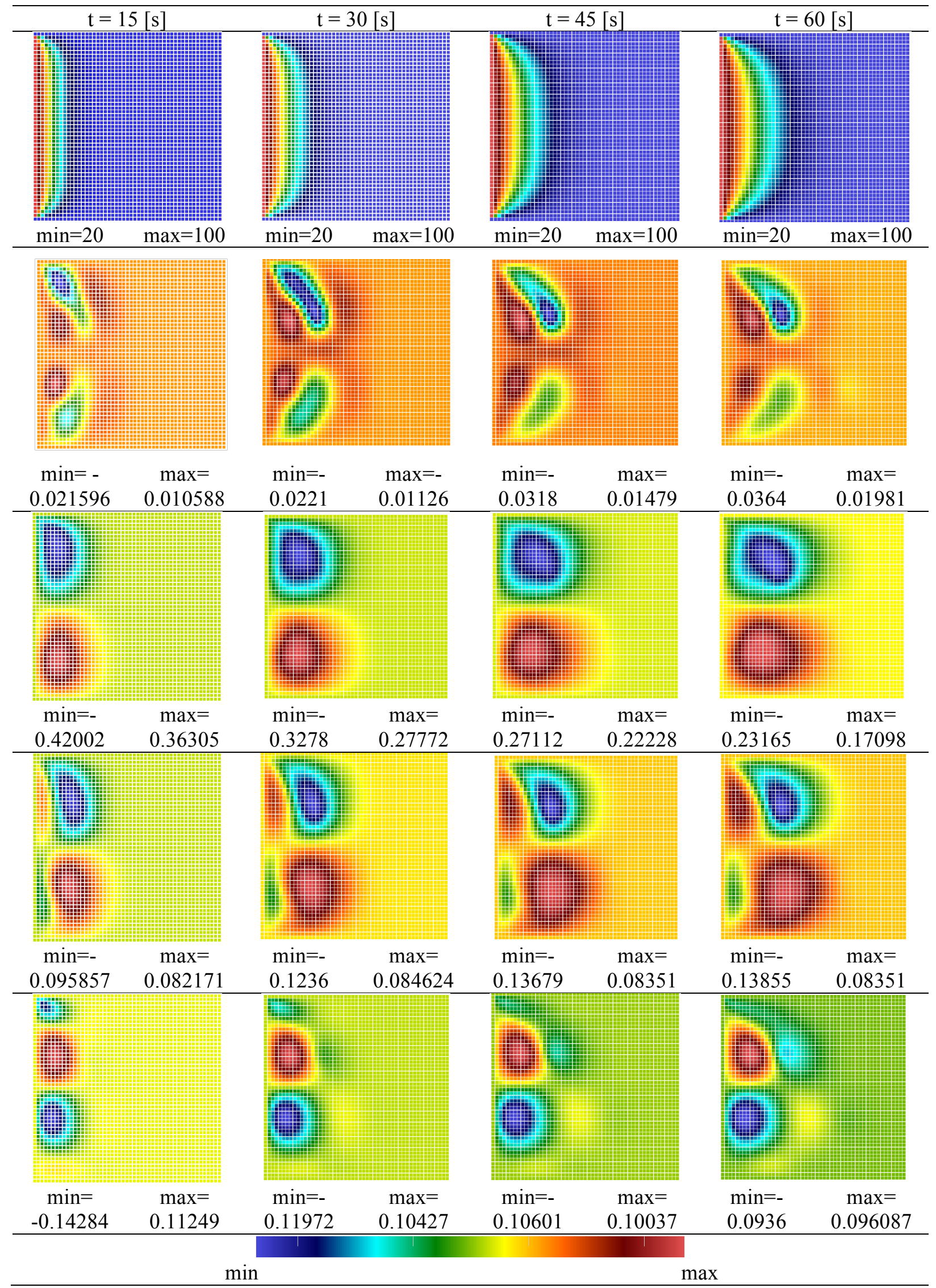


Table 2. Deterministic and stochastic solutions for the Neumann boundary heat problem.

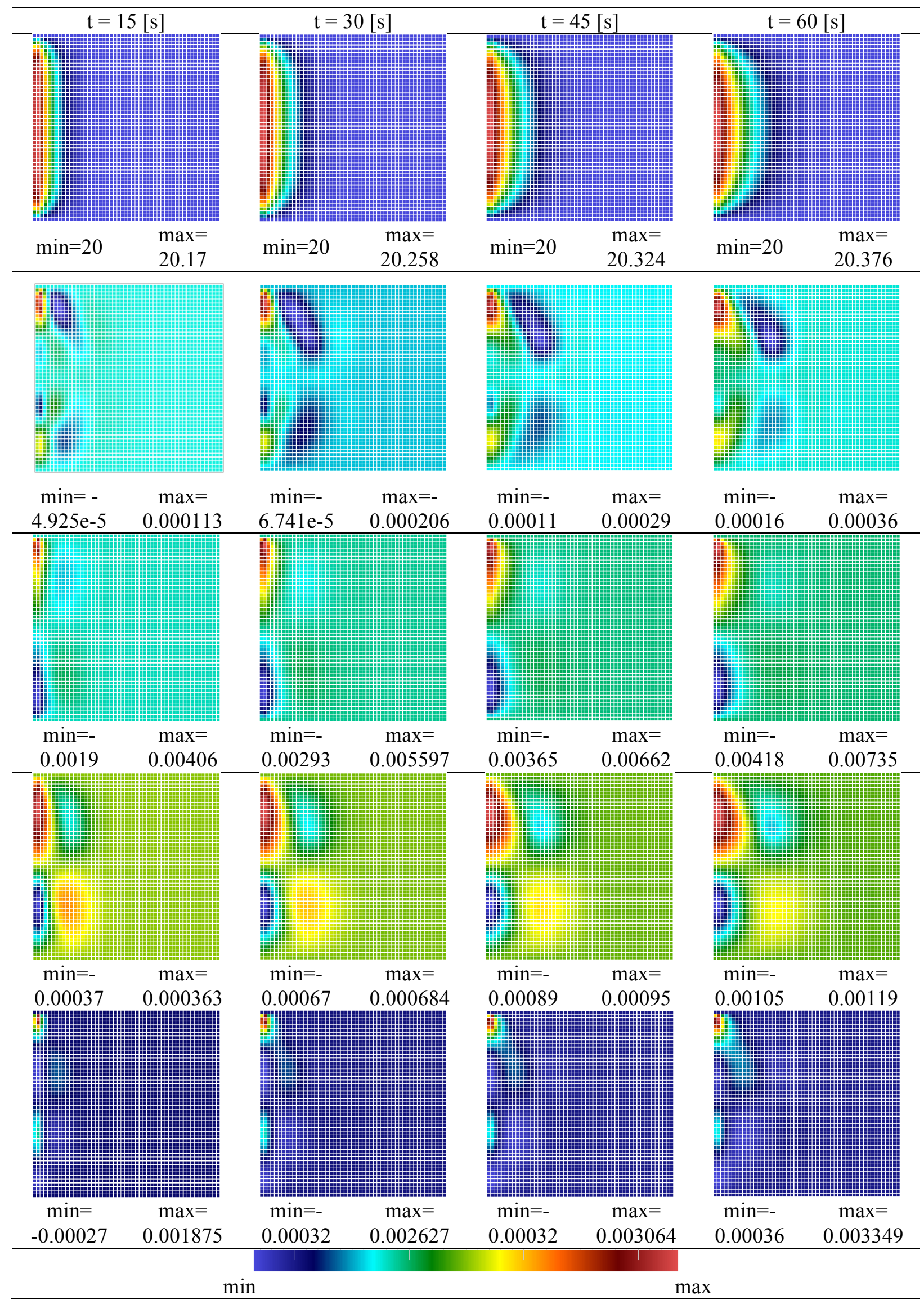




\section{CONCLUSIONS}

This work presents a useful form to simulate a heat transfer process with a random heat conduction coefficient, which is the first step to develop a stochastic moving particle model that simulates an energy coupled Navier-Stokes problem by a Lagrangian scheme, this becomes important for a reliability design in high deformation, high Reynolds and multiphase problems.

One of the mayor advantages for the reliability design in the SSSPH method is the capability to solve more than one stochastic term that usually has different behavior. Adding a Karhunen-Loeve expansion in every desired property could represent diverse stochastic behaviors and relationships among them.

Although the heat conduction problem has a very stable solution in a lot of methods, the implementation in SPH shows minor instabilities that are similar in all the stochastic modes. Including the stochastic spectral scheme in the SPH method disrupts the solution, so an artificial diffusion term is implemented to obtain adequate results and reduce the instabilities.

The SSSPH method keeps the same disadvantages of the current spectral stochastic methods about the global domain shape; the use of a symmetric square domain is a restriction because of the covariance input functions.

\section{REFERENCES}

[1] G. Stefanou, "The stochastic finite element method: Past, present and future," Computer Methods in Applied Mechanics and Engineering, vol. 198, no. 9-12, pp. 1031-1051, Feb. 2009.

[2] S.-K. Choi, R. V. Grandhi, and R. A. Canfield, Reliability-based Structural Design. .

[3] G. Lin, X. Wan, C.-H. Su, and G. E. Karniadakis, "Stochastic Computational Fluid Mechanics," pp. 21-29, 2007.

[4] V. A. B. Narayanan and N. Zabaras, "A spectral stochastic finite element implementation of probabilistic advective-diffusive transport with stabilization based on multiscale phenomena," no. May 2003.

[5] D. Ghosh and G. Iaccarino, "Applicability of the spectral stochastic finite element method in time-dependent uncertain problems," vol. 2, pp. 133-141, 2007.

[6] H. C. Elman, O. G. Ernst, D. P. Oõleary, and M. Stewart, "Efficient iterative algorithms for the stochastic finite element method with application to acoustic scattering," vol. 194, pp. 1037-1055, 2005.

[7] S. Li and W. K. Liu, Meshfree particle methods, vol. 25, no. 2-3. 2000, pp. 99-101.

[8] J. J. Monaghan, "Smoothed particle hydrodynamics," Reports on Progress in Physics, vol. 68, no. 8, pp. 1703-1759, Aug. 2005.

[9] G. R. Liu and M. B. Liu, Smoothed Particle Hydrodynamics: A Meshfree Particle Method. 2003, p. 449.

[10] R. Capuzzo-Dolcetta, "A criterion for the choice of the interpolation kernel in smoothed particle hydrodynamics," Applied Numerical Mathematics, vol. 34, no. 4, pp. 363-371, Aug. 2000.

[11] S. S. Observatories, "Kernel Estimates as a Basis for General Particle Methods in Hydrodynamics," vol. 453, pp. 429-453, 1982. 
[12] B. Sudret and A. Der Kiureghian, "Stochastic Finite Element Methods and Reliability A State-of-the-Art Report," no. November, 2000.

[13] S. K. Sachdeva, P. B. Nair, and A. J. Keane, "Comparative study of projection schemes for stochastic finite element analysis," Computer Methods in Applied Mechanics and Engineering, vol. 195, no. 19-22, pp. 2371-2392, Apr. 2006.

[14] S. Huang, S. Mahadevan, and R. Rebba, "Collocation-based stochastic finite element analysis for random field problems," Probabilistic Engineering Mechanics, vol. 22, no. 2, pp. 194-205, Apr. 2007.

[15] R. Ghanem, "Polynomial Chaos in Stochastic Finite Elements," no. 89, 1990.

[16] R. Ghanem, "Ingredients for a general purpose stochastic finite elements implementation," vol. 7825, no. 98, 1999.

[17] M. B. Liu and G. R. Liu, "Smoothed Particle Hydrodynamics ( SPH ): an Overview and Recent Developments," Methods, pp. 25-76, 2010.

[18] J. J. Monaghan, "Smoothed Particle Hydrodynamics and Its Diverse Applications," Annual Review of Fluid Mechanics, vol. 44, no. 1, pp. 323-346, Jan. 2012.

[19] P. Cleary, "Conduction Modelling Using Smoothed Particle Hydrodynamics," Journal of Computational Physics, vol. 148, no. 1, pp. 227-264, Jan. 1999.

[20] D. J. Price, "Modelling discontinuities and Kelvin-Helmholtz instabilities in SPH," no. August 2008.

[21] J. J. Monaghan, "On the problem of penetration in particle methods," Journal of Computational Physics, vol. 82, no. 1, pp. 1-15, May 1989. 